\title{
Development of a Drowsiness Warning System based on the Fuzzy Logic
}

\author{
Nidhi Sharma \\ M.Tech Scholar, ACET \\ Amritsar, Punjab, INDIA
}

\author{
Prof. V. K. Banga \\ HOD(ECE/EEE),ACET \\ Amritsar,Punjab,INDIA
}

\begin{abstract}
Nowadays, driving support systems, such as car navigation systems, are getting common, and they support drivers in several aspects. It is important for driving support systems to detect status of driver's consciousness. Particularly, detecting driver's drowsiness could prevent drivers from collisions caused by drowsy driving. In this paper, we discuss a system and a method for detecting driver's drowsiness, which uses the Image processing and Fuzzy logic to detect Driver's drowsiness.
\end{abstract}

\section{General Terms}

Image processing,Fuzzylogic segmentation binary image algorithm

\section{Keywords}

Drowsiness warning system; Vehicle safety; Image processing; Fuzzy logic inference

\section{INTRODUCTION}

Driver drowsiness is an important factor in the motoring of vehicle accidents $[19,8,2,1]$. It was demonstrated that driving performance deteriorates with increased drowsiness with resulting crashes constituting more than $20 \%$ of all vehicle accidents [13].Traditionally transportation system is no longer sufficient. Recently artificial intelligence techniques has emerged and became a popular topic among transportation researchers In recent years, there has been growing interest in intelligent vehicles. A notable initiative on intelligent vehicles was reported by the U.S. Department of Transportation with the mission of prevention of highway crashes [12]. The ongoing intelligent vehicle research will revolutionize the way vehicles and drivers interact in the future. The US National Highway Traffic Safety Administration estimates that in the US alone approximately 100,000 crashes each year are caused primarily by driver drowsiness or fatigue. Thus incorporating automatic driver fatigue detection mechanism into vehicles may help prevent many accidents. One can use a number of different techniques for analyzing driver exhaustion. One set of techniques places sensors on standard vehicle components, e.g., steering wheel, gas pedal, and analyzes the signals sent by these sensors to detect drowsiness [7, 9]. It is important for such techniques to be adapted to the driver. A second set of techniques focuses on measurement of Physiological signals such as heart rate, pulse rate, and Electroencephalography (EEG) [8]. It has been reported by researchers that as the alertness level decreases EEG power of the alpha and theta bands increase. Hence providing Indications of drowsiness. However this method has drawbacks in terms of practicality since it requires a person to wear an EEG cap while driving. A third set of solutions focuses on computer vision systems that can detect and recognize the facial motion and appearance changes occurring during drowsiness $[4,11,16]$.The advantage of computer vision techniques is that they are non-invasive, and thus are more amenable to use by the general public. There are some significant previous studies about drowsiness detection using computer vision techniques. Most of the published research on computer vision approaches to detection of fatigue has been focused on the analysis of blinks and head movements[13,15].However, in the fatigue detection systems developed to date, drowsiness warning system using image processing has become most widely used because it provides a remote detection[13].

Due to the increase in the amount of automobile in recent years, problems created by accidents have become more complex as well. Traditional transportation system is no higher sufficient. In recent years, the intelligent vehicle system has emerged and became a popular topic among transportation researchers. However, the research of safety in vehicle is an important subset of intelligent vehicle system research. Meantime, active warning system is one of the designs on active safety system. The safety warning systems, mostly active warning systems for preventing traffic accidents have been attracting researchers.

Owing to the progress of digital signal processing technology, real time image processing is beginning to be achieved breakthroughs in the field of many practical applications. Typically, after high hours of driving or in absent of alert mental state, the eyelids of driver will become heavy due to fatigue. The attention of driver starts to lose focus, and that creates risks for accidents. These are typical reactions of fatigue, which is very dangerous. Usually many exhausted drivers are not aware that they are in falling asleep. In fact, many such drivers can fall asleep any time during their driving. In an image fatigue detection, correct and real time decision is important, a fuzzy logic determined algorithm is proposed to determine the level of fatigueness and then warn the driver accordingly.

\section{DROWSINESS WARNING SYSTEM}

Owing to the great improvement on microprocessor in recent years a large, two-dimensional image can be easily process by a computer. The image analysis techniques have been greatly accepted and applied. In the proposed method, A camera is installed on the front near the back mirror glasses for taking consecutive facial images of the driver .It then uses program which is written in matlab code to detect the position of eyes based on the images taken. Finally, a fuzzy logic is used to determine the driver's alertness.

The algorithm for this system is as shown below: 


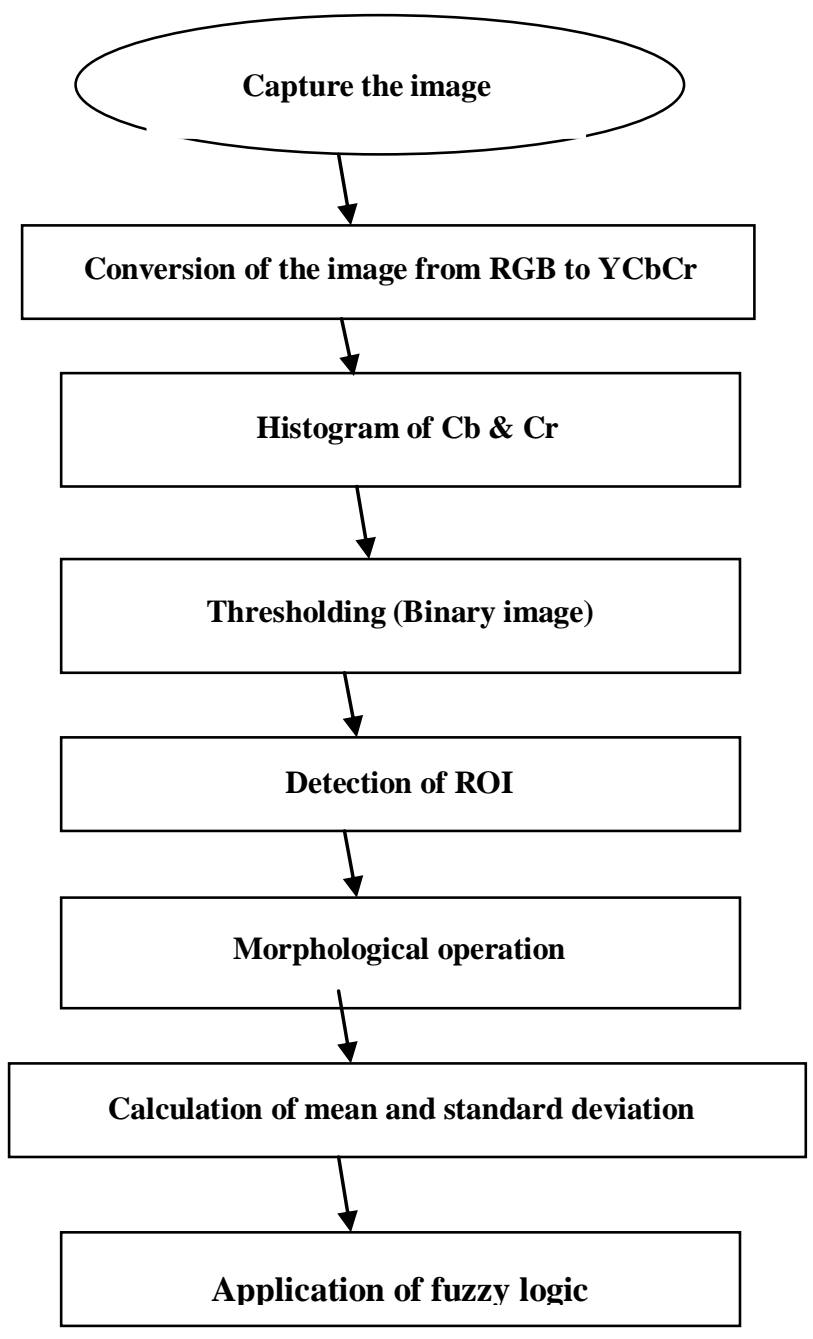

Fig.2.1 showing the algorithm

\section{IMAGE PROCESSING FOR THE DETECTION OF EYE}

This section explains the various steps involved in the detection of ROI implementing the image processing.

\subsection{Capturing the image}

An image which taken inside a vehicle includes the driver driver's face. Typically a camera takes images within the RGB model (Red, Green and Blue). However, the RGB model includes brightness in addition to the colours. When it comes to human's eyes, different brightness for the same colour means different colour. When analyzing a human face, RGB model is very sensitive in image brightness. Therefore, to remove the brightness from the images is second step. We use the $\mathrm{YCbCr}$ space since it is widely used in video compression standards. Since the skin-tone colour depends on luminance, we nonlinearly transform the $\mathrm{YCbCr}$ colour space to make the skin cluster luma-independent. This also enables robust detection of dark and light skin tone colours. The main advantage of converting the image to the $\mathrm{YCbCr}$ domain is that influence of luminosity can be removed during our image processing. In the
RGB domain, each component of the picture (red, green and blue) has a different brightness. However, in the $\mathrm{YCbCr}$ domain all information about the brightness is given by the $\mathrm{Y}$ component, since the $\mathrm{Cb}$ (blue) and $\mathrm{Cr}$ (red) components are independent from the luminosity. The following conversions are used to segment the RGB image into $\mathrm{Y}, \mathrm{Cb}$ and $\mathrm{Cr}$ components:

$\mathrm{Cr}=0.439 * \mathrm{R}-0.368 * \mathrm{G}-0.071 * \mathrm{~B}+128$

$\mathrm{Cb}=0.148 * \mathrm{R}-0.291 * \mathrm{G}-0.439 * \mathrm{~B}+128$

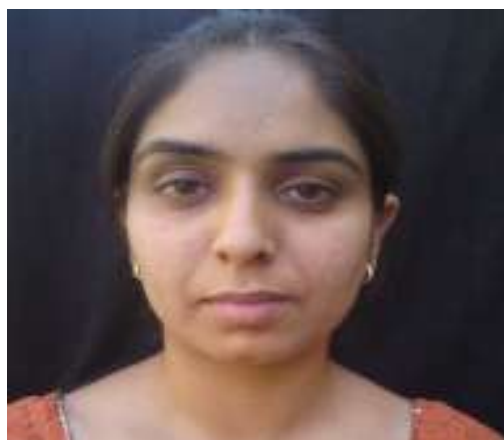

Fig.3.1.1: showing image in RGB

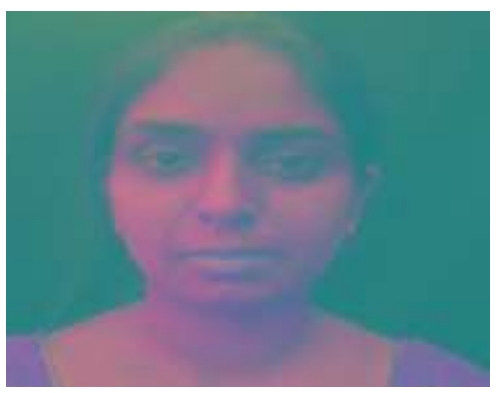

Fig. 3.1.2: showing image in $\mathrm{YCbCr}$

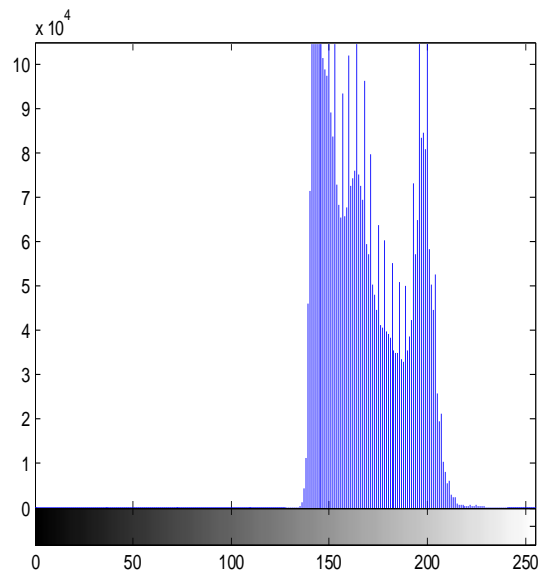

Fig.3.1.3: Showing plot of cb 


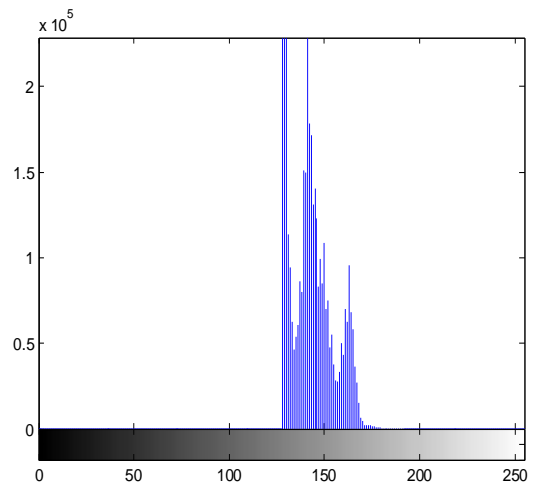

Fig..3.1.4: Showing plot of cr

\subsection{Binary Image Processing and Segmentato -n of the Image}

The $\mathrm{Cb}$ and $\mathrm{Cr}$ components give a good indication on whether a pixel is a part of the skin or not is to reject regions in the image that are not faces The next step is to use binary image processing to create clearer delineations in these regions. The algorithm was implemented in MATLAB. Faces can be distinguished by applying maximum and minimum threshold values for both $\mathrm{Cb}$ and $\mathrm{Cr}$ components. The thresholds that were chosen based on the histograms are as following:

$130<=C r<=155$

$159<=C b<=230$

Narrowing down these thresholds increases the probability that the accepted pixels are actually part of the skin. Based on the $\mathrm{Cb}$ and $\mathrm{Cr}$ thresholding, a resulting black and white "mask" is obtained.

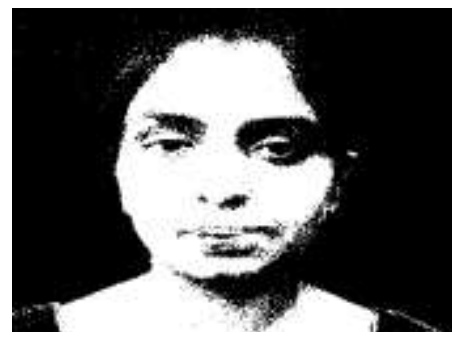

Fig:3.2.1: showing binary image of the face with open eyes

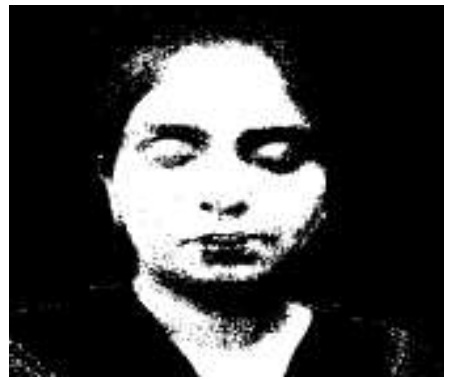

Fig.3.2.2: showing binary image of the face with closed eyes

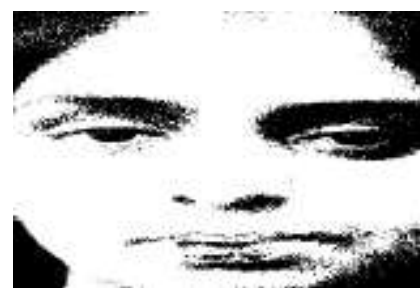

Fig.3.2.3: showing segmented image of the face with open eyes

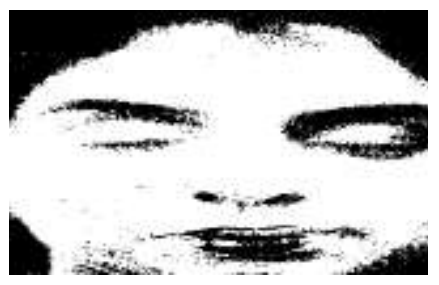

Fig.3.2.4: showing segmented image of the face with closed eyes

\section{EYE REGION DETECTION}

Since we are taking only the face area, we know that the region of interest is the region containing the eyes. We divided the face into four quadrants, the region of eyes will be uppermost two quadrants Since blinking of the eyes usually happen concurrently, we can then assume that the right eye will be positioned at the upper left-hand side of the face. Therefore, calculation will be based on only one eye. By taking these assumptions, the search for the eye will be limited to the area this limited area will make the search more efficient .The
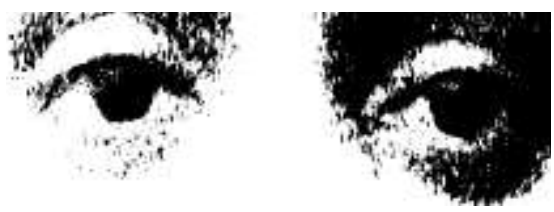

Fig.4.1: showing the ROI of the open eyes
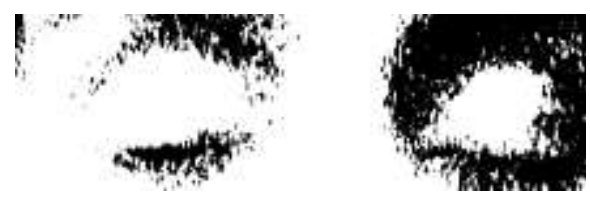

Fig.4.2: showing the ROI of the closed eyes

following Fig. shows the region of face containing eyes.From this region we will be taking only one eye for our calculations.following Fig. shows the eye detected. 


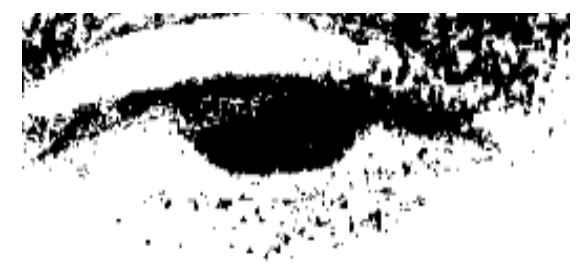

Fig.4.3: showing the eye

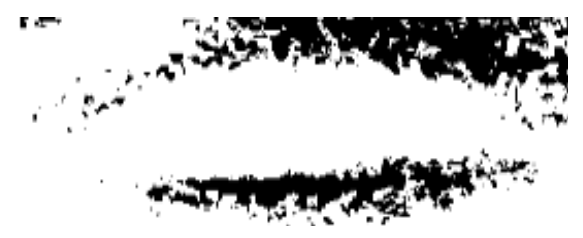

Fig.4.4: showing the eye

\section{MORPHOLOGICAL OPERATIONS ON} THE EYE

This eye is then refined through binary morphological operations to reduce the background contribution and remove holes within eye region. The image is first eroded with a pixel face-shaped kernel to eliminate small background objects. This eroded image is then dilated with a pixel face-shaped kernel to refill gaps if any.

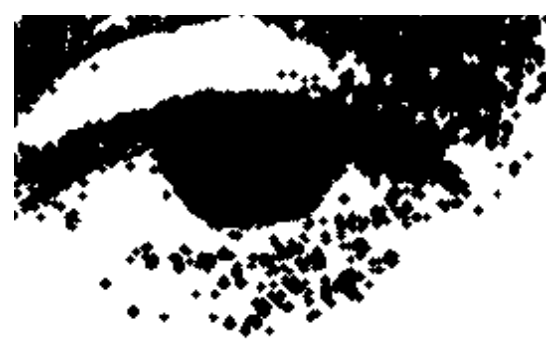

Fig.5.1: showing the eye after morphological erosion

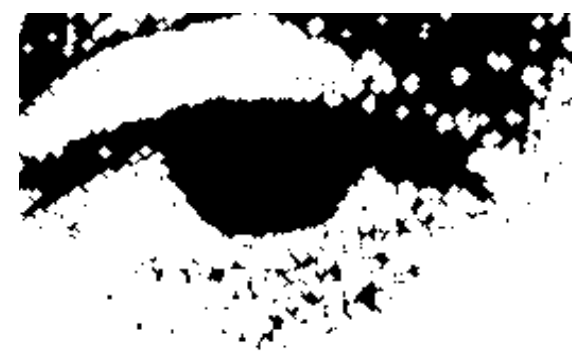

Fig.5.2: showing the eye after morphological dilation

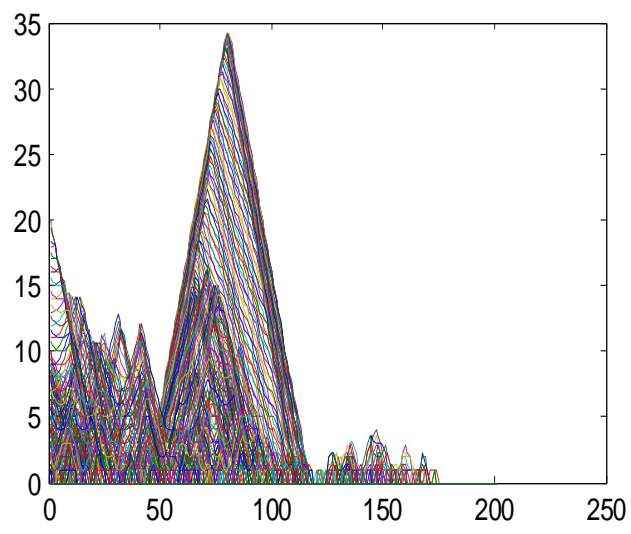

Fig. 5.3: showing the plot of the open eye

This plot shows that when the eyes are open high peak pertaining to black cornea will be obtained corresponding to the position of the cornea. Whereas for closed eye the plot is a valley

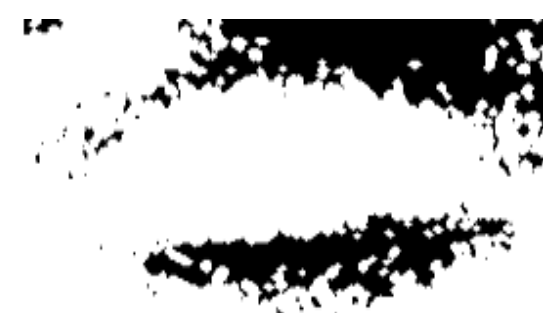

Fig.5.4: showing the eye after morphological erosion

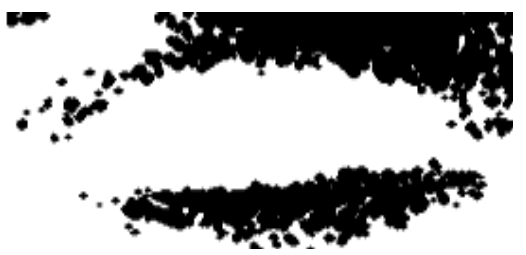

Fig.5.5: showing the eye after morphological dilation

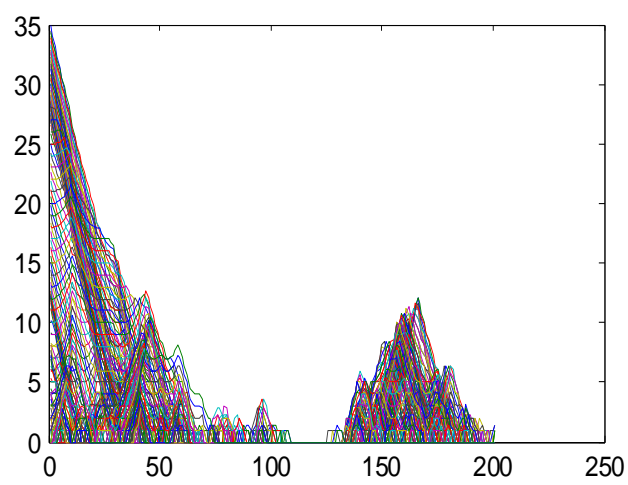

Fig.5.6: showing the plot of the closed eye 


\subsection{Detecting the openness of eyes:}

In this section, the concept of threshold is used to detect the openness of the driver's eyes. Thresholding is the operation of setting a pixel to black if the value is below a given threshold. On the other hand, a pixel is set to white if the value is above the threshold. From this process, we can generate a black-and-white image.. After obtaining the thresholding value based on set the skin pixel to white and the eye to black. Based on the number of black pixels in an image, we are able to determine whether the eye is open or closed.

\section{METHOD OF DROWSINESS LEVEL ESTIMATION}

This section explains the implementation of Fuzzy logic in determining the level of drowsiness.

\subsection{Fuzzy logic:}

The concept of fuzzy set is a class with unsharp boundaries. It provides a basis for a qualitative approach to the analysis of complex systems in which linguistic rather than numerical variables are employed to describe system behaviour and performance. In this way, a much better understanding of how to deal with uncertainty may be achieved, and better models of human reasoning may be constructed. The concept of fuzzy logic has gained wide acceptance in recent years and have found numerous applications in expert systems and artificial intelligence applications. Fatigue is a type of fuzzy bodily state. It cannot be quantified objectively. Thus, we use computers to apply the fuzzy logic and determine the level of fatigueness. The variable used by the drowsiness detection system encompasses the blinking time and the mean of the eye. The risk factor is calculated based on rule-table decision. The standard deviation and mean are taken as the input variables. The physical state of the eye is the output variable.

\subsection{Establishing the grade of membeship}

To establish the grade of membership, we enter the standard deviation and mean of the eye as the input variables.

Low standard deviation Triangular $[0 ; 0 ; 0.1]$

Medium standard deviation Triangular $[0.75 ; 1.5 ; 3.75]$

High standard deviation Triangular $[3 ; 6 ; 6]$

Low mean of the eye Triangular $[0 ; 0 ; 0.75]$

Medium mean of the eye Triangular $[0.5 ; 1.125 ; 1.5]$

High mean of the eye Triangular [1.25;3;3]

The physical state of the driver as the output:

close Triangular $[0 ; 0 ; 0.4]$

drowsy Triangular $[0.3 ; 0.5 ; 0.8]$

open Triangular $[0.6 ; 1 ; 1]$

Fig.6.2.1 is the input variable, standard deviation. Fig.6.2.2is the input variable, mean of the eye, and Fig. 6.2.3 is the output variable, physical state of the eyes, membership function. The variable is changed according to the driver's physical conditions. For example, when the driver is tired, his eyes will involuntarily extend the mean of the eye. When the driver is alert, his standard deviation is low and his mean of the eye will also be low. However, when the driver is exhausted, his standard deviation is high and his mean of the eye will also be higher. Therefore, based on standard deviation and mean of the eye, nine rule base of the fuzzy logic is defined as follows.
Rule 1: IF standard deviation is low AND mean is also low THEN the eyes will be open

Rule 2: If standard deviation is low AND mean is medium THEN the eyes will be open

Rule 3: IF standard deviation is low AND mean is high THEN the eyes will be drowsy

Rule 4: IF standard deviation is medium AND mean is low THEN the eyes will be drowsy

Rule 5: IF standard deviation is medium AND mean is medium THEN the eyes will be drowsy

Rule 6: IF standard deviation is medium AND mean of the eye is high THEN eyes will be closed

Rule 7: IF standard deviation is high AND mean of the eye is low THEN the eyes will be drowsy

Rule 8: IF standard deviation is high AND mean of the eye is medium THEN the eyes will be closed

Rule 9: IF standard deviation is high AND mean of the eye is high THEN closed

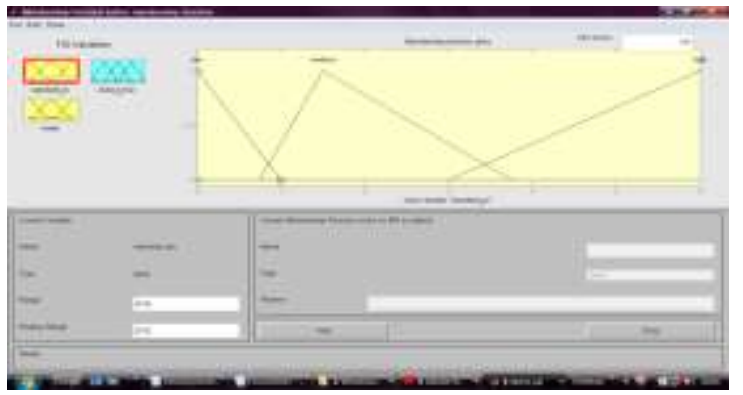

Fig.6.2.1: showing degree of membership of the standard deviation

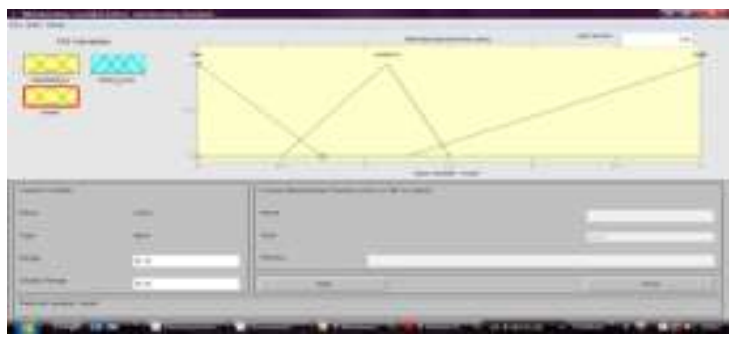

Fig.6.2.2: showing degree of membership of the mean

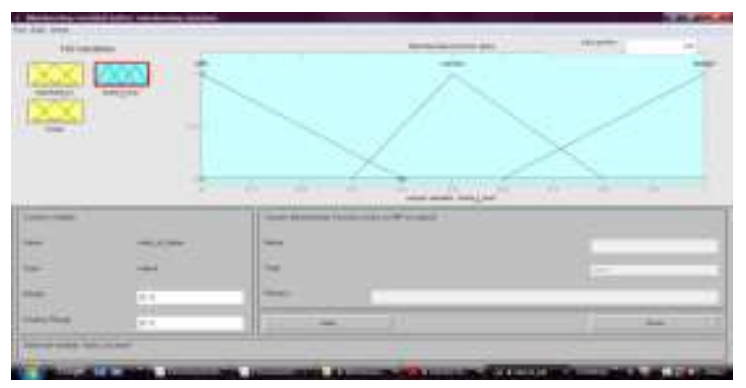

Fig.6.2.3: showing the degree of membership of the physical state of the driver 


\subsection{Fuzzification:}

Fuzzification is related to the linguistic terms. It is a process of converting measurable values into subjective judgements. The inputs of proposed system are the standard deviation and mean of the eye region. These two variables are converted into "low value of standard deviation", "medium value of standard deviation", "high value of standard deviation", "low value of the mean", "medium value of the mean, and "high value of the mean" based on the images taken. Membership functions of the input and output variables

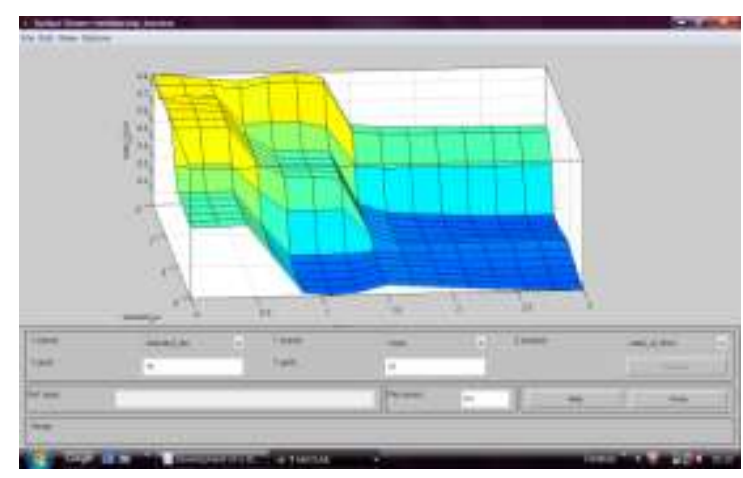

Fig. 6.3.1: showing the fuzzy surface view

\section{CONCLUSION:}

A vehicle driver drowsiness warning system using fuzzy logic and image processing technique is used in this method. However, one important issue that is not addressed in this paper, the lack of lighting during sunset may cause errors when the images are read. Also, it may be too dark at night for the system to properly detect the driver's eyes more over this method finds its limitation in detecting the eyes of a person wearing spectacles. In future work Infrared light could be a solution for taking the images and thus lower the possible errors caused of the lack of or change of the lighting.

\section{REFERENCES}

[1] Azim Eskandarian, Member, IEEE and Ali Mortazavi (2007)."Evaluation of a Smart Algorithm for Comme-rcial Vehicle Driver Drowsiness Detection." IEEE Intelligent Vehicles Symposium Istanbul,Turkey, June 13-15

[2] Eriksson, M and Papanikolopoulos, N.P(1997) "EyeTracking for Detection of Driver Fatigue", IEEE Intelligent Transport System Proceedings, 314-319.

[3] Fukuda, J.Akutsu, E., \& Aoki, K. (1995).” Anestimation of driver's drowsiness level using Keeping". JSAE Review, 16, 185-199.

[4 ] Face Detection Using Colour Thresholding and Eigen Image Template Matching Diedrick Marius,Sumita Pennathur and Klint Rose.

[5] Haisong Gu, Yongmian Zhang, and Qiang Ji, (2005). "Task oriented facial behaviour recognition with selective sensing, Compute. Vis. Image Understat”. vol. 100, no.3, 385415 .
[6] Kim Hong, Chung (2005)“"Electroencephalogram-raphic study of drowsiness in simulated driving with Sleep deprivation". International Journal of Industrial Ergonomics, Volume 35, Issue 4, April 2005, 307-320.

[7] K. Harimast (2002) "Human Maehinc. Intedae in an Intelligent vehicle" SAU.vol.56, 4-7.

[8] L.Barr, H.Howrah, S.Popkin, RJ.Carrol 1(2009) “ A review and evaluation of emerging driver fatigue detection, measures and technologies", A Report of US department of transportation Washington DC.

[9] Lin, S. H., Kung, S. Y., \& Lin, L. J. (1997). "Face recognition/detection by probabilistic decision-based neural network."IEEE Transactions on Neural Networks, 8(1), 114-132

[10] L. Sirovich and M. Kirby (1987). "Low-dimensional procedure for the characterization of human faces", Journal of Optical Society of America, vol. 4, 519.

[11] Mai Suzuki, Nozomi Yamamoto, Osami Yamamoto, Tomoaki Nakano and Shin Yamamoto (2006). "Measurement of Driver's Consciousness by Image Processing-A Method for Presuming Driver's Drowsiness by Eye-Blinks coping with Individual Differences". IEEE International Conference on Systems, Man, and Cybernetics,October 8-11 Taipei, Taiwan.

[12] NHTSA (2009), "Drowsy driver's detection and warning system for commercial vehicle drivers: Field proportional test design, analysis, and progress "National Highway Traffic Safety Administration, Washington, DC- June 2009.

[13] Perez, C. A., Palma, A., Holzmann, C. A., \& Pena (2001). "Face and eye tracking algorithm based on digital image processing”. IEEE International Conference, 2 .1178-1183.

[14] P.P. Caffier, U. Erdmann, and P. Ullsperger (2003) "Experimental evaluation of eye-blink parameters as a Drowsiness measure”, Eur J Appl Physiol, 89(3-4). 319-325.

[15] S.Singh. N.P.Fapanikolopaulas (1999) "Monitoring Driver Fatigue Using Facial Analysis Technologies". Proceedings of IEEE international conference on the Intelligent Transportation.

[16] Seki, M., Shimotani, M., \& Nishida, M. (1998). “A study of blink detection using bright pupils". JSAE Review, 19. 4967.

[17] Sugiyama, K., Nakano, T., Yamamoto, S., Ishihara, T., Fujii, H., \& Akutsu, E. (1996). "Method of detecting drowsiness level by utilizing blinking duration". JSAE Paper 9630273.

[18] Ueno, H. Kaneda \& Tasukino (1994). "Development of drowsiness detection system". In Vehicle navigation and information system conference. 15-20. 